ITC $3 / 50$

Information Technology and Control

Vol. 50 / No. 3 / 2021

pp. 546-557

DOI 10.5755/j01.itc.50.3.28672
An Optimized Traffic Grooming Through Modified PSO Based Iterative Hungarian Algorithm in Optical Networks

Received 2021/03/17

Accepted after revision 2021/08/04

HOW TO CITE: Kumarnath, J., Batri, K. (2021). An Optimized Traffic Grooming Through Modified PSO Based Iterative Hungarian Algorithm in Optical Networks. Information Technology and Control, 50(3), 546-55\%. https://doi.org/10.5755/j01.itc.50.3.28672

\title{
An Optimized Traffic Grooming Through Modified PSO Based Iterative Hungarian Algorithm in Optical Networks
}

\section{J. Kumarnath}

Assistant Professor, Department of ECE, PSNA College of Engineering and Technology, Dindigul, Tamilnadu, India; e-mail: jkumarnath@psnacet.edu.in

\section{K. Batri}

Professor, Department of ECE, PSNA College of Engineering and Technology, Dindigul, Tamilnadu, India; e-mail:krishnan.batri@psnacet.edu.in

Corresponding author: jkumarnath@gmail.com

Due to huge size of the data and quick transmission of data between the nodes present in the optical network, a condition known as network traffic is created among the nodes of the network. This issue of traffic can be overcome by employing numerous traffic grooming techniques. This research work focuses on the usage of multi objective modified Particle Swarm Optimization (PSO) algorithm for determining the best suitable shortest path and discusses an innovative visibility graph based Iterative Hungarian Traffic grooming algorithm which is implemented to reduce the blocking ratio by improving the allocation of bandwidth between the users. The multi-objective parameters such as available wavelength, throughput and all-inclusive delay have been taken into consideration for determining the optimal path in the routing scheme. The formulation implemented with the modified PSO based Iterative Hungarian approach focusses on grooming the traffic thereby optimal throughput and least Blocking Probability are achieved. Then, the performance analysis is carried out by means of performance measures such as traffic throughput, transceivers count, average propagation delay, blocking ratio, and success ratio. It can be inferred that the proposed work obtains enhanced outcomes when they are compared to the other existing techniques.

KEYWORDS: Optical Network, Traffic Grooming, Multi Objective modified Particle Swarm Optimization (PSO) algorithm, Visibility Graph based Iterative Hungarian Traffic grooming algorithm, Allocation of Bandwidth. 


\section{Introduction}

Optical Networks incorporate the use of light signal to transmit information to various categories of telecommunications networks. By adapting the optical switching separately for unicast, multicast and incast using optical grooming, the load in a network can be reduced. Traffic grooming occurs in a network due to the following factors: the large size of the data present in the network and also the rapid transmission of data within the network. Almost all types of networks are likely to face this problem of traffic which occurs within the network. Mostly, the traffic in the Data Center Networks (DCNs) which is emerging as the significant problem that has gained more attention in recent times [17]. The resource utilization focus on bandwidth and energy proportionality is addressed and the power consumption of the network is also enhanced by employing the power settings in the Optical Network architectures [2]. To cope with the current trend in the telecommunication system, the evolution of traffic demands within the network has been improved in recent times. Thanks to the intervention of applications in this realm, that an exponential growth rate is predicted in the traffic demand especially by using applications like real-time and high-definition video communication in a distributed environment owing to the rapid development of technology in time by time [10]. The data transportation is enhanced up to a certain extent by employing an advanced type of a lightpath by means of light trail in the fixed wavelength grid network. Wavelength division multiplexing (WDM) is a technique that transmits the number of optical signals carried on a single fiber using different wavelengths. WDM systems are popular over telecommunication, because they allow them to maximize network capacity without burdening the use of lot of fiber. The elastic optical mesh network is an advanced form of Wavelength Division Multiplexing (WDM) optical mesh network which replaces the WDM optical mesh network as the WDM optical mesh network might become outdated.

Multiplexing of the Optical Orthogonal Frequency Division (O-OFDM) [4] is a hopeful technique where the traffic data are loaded on spectrum overlapping subcarriers and multiple orthogonal to attain the efficacy of spectrum highly. Afterwards the bandwidth-variable transponders (BVT) alongside the adjustable central frequency can handle various traffic links elastically by producing a suitable number of subcarriers in O-OFDM. The lightpath can be shared by a light trail route through communication channel using optical time division multiplexing (OTDM) technology. A node can access flexibly the allocated time slot till the time slot is not utilized by the upstream node. To meet the emerging need and growth of Internet data, elastic optical networks (EONs) have recently emerged as an effective solution that can adapt to real-time and data transfer capabilities and thus effectively manage network resources. In Elastic Optical Network (EON), multiple consecutive slots of the spectrum could be assigned to one link instead of a single wavelength stream. EON therefore needs more complex algorithms for the allocation of spectrum resources than conventional WDM networks, and can also be known as algorithms for routing and spectrum allocation (RSA). Traffic Grooming incorporates into the optical Network for efficient multiplexing/ demultiplexing and switching of low-speed streams to/from high-bandwidth channel. The bandwidth can be used efficiently and flexibly for its communication channel accessed by intermediate nodes in the lightpath. Thus, Traffic Grooming enables network building and provision of resources that can qualify cost-effective use of both network bandwidth and electronic switching. Figure 1(a) represents allocation of spectrum based on convention WDM optical Network and Figure 1(b) represents the allocation of spectrum with traffic grooming. Figure 1(b) shows the advantage of multiplexing the traffic incorporating the efficient utilization of spectrum.

\section{Figure 1}

Traffic grooming in OFDM based EON (a) Allocation of spectrum without traffic grooming, (b) Allocation of spectrum by traffic grooming

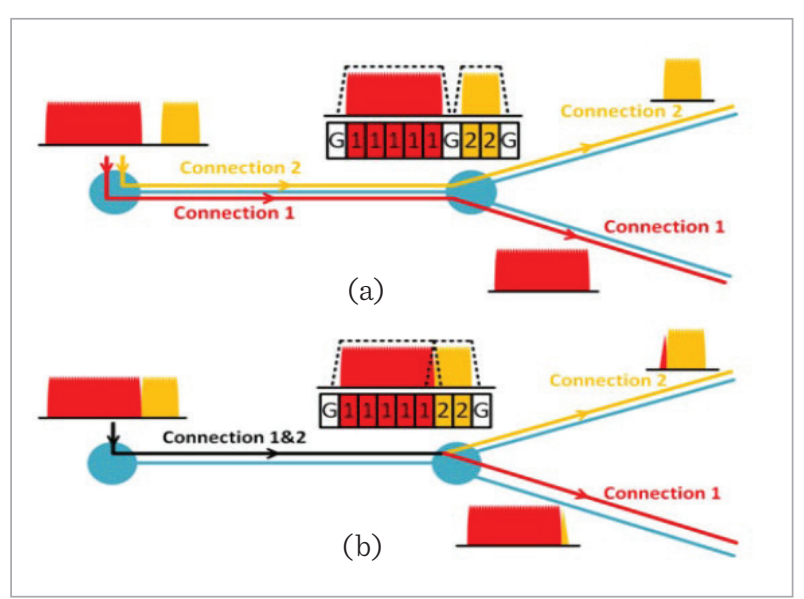




\subsection{Objectives}

The objectives involved in the research work are mentioned below:

- To detect the path failures and the shortest paths among the nodes by Multi objective modified PSO.

- To find the availability of bandwidth of the specified path for requested users by the Visibility Graph based Iterative Hungarian Traffic Grooming algorithm.

- To reduce the traffic congestion request blocking ratio in a system of network by allocation of bandwidths.

\subsection{Paper Organization}

Paper organization is described in this section. Section 2 enlists the literature review part where the research works related to the traffic problem in networks are briefly listed one by one. Section 3 marks the proposed work of Iterative Hungarian Traffic Grooming based on the Visibility graph method which reduces the blocking ratio by improving the allocation of bandwidth between the users. Section 4 analyzes the execution of the proposed method by interrelating obtained results with the existing ones. Then finally the efficiency of the proposed work and the overall conclusion of the research work are provided in the last section.

\section{Related Works}

Kaur and Rattan [5] optimized the traffic grooming problem existing in the optical network with the aid of the Wind Driven Optimization (WDO) algorithm. The information about the variables, types, objective functions, parameters and the constraints related to the traffic grooming problem were also discussed in the paper. An innovative approach of auxiliary graph model [8] was developed which possessed the ability to reflect every characteristic present in a path of light and also with reduced shortcomings. Hence, two different and efficient traffic grooming methods such as $\mathrm{Bw}-\mathrm{Tx} / \mathrm{Rx}$ and $\mathrm{O} / \mathrm{E} / \mathrm{O}$ algorithms were proposed in the research work based on an auxiliary graph model. The Multiple Multi-hop noncontiguous elastic lightpath algorithm was proposed in which the required spectrum of traffic demand was divided into different granular spectrum depending on existing availability and classification status of spectrum slots thereby preserving Spectrum Continuity Constraint [9].

Panayiotou et al. utilized an innovative traffic grooming approach [12] based on the principle of grooming or routing the multicast calls in the hybrid graphs (HGs) for investigating the issue of traffic grooming prevailing in the optical networks. Depending upon the incoming of each and every multicast call, the grooming or routing is carried out in HGs by implementing a novel heuristic hybrid Steiner tree approach. The use of bandwidth resources was improved by employing a very suitable traffic grooming approach in the dynamic optic networks [24]. The issue of traffic grooming existing under dynamic traffic circumstances in a Spatial Division Multiplexing Enabled Elastic Optical Networks (SDM-EON) which consisted of multi core fibers were solved by making use of a specially designed Auxiliary Graph (AG).

A novel structure for optimizing the multi period planning scenarios for the optical networks that were enabled by SBVT was proposed in [3]. The Sliceable Bandwidth Variable Transponder (SBVT) was one of the transmission modules that enabled the line rates to adapt simultaneously with the assured multi directionality and hence this module was considered to be a step forward in the technology of optical transport. An innovative approach of three-layered auxiliary graph (AG) for addressing the traffic grooming problem present in a mixed electrical-optical network was presented based on the scenario of dynamic traffic [22]. A significant issue of coordinating the optical and electrical traffic grooming when employing the new connection request in Sliceable Bandwidth-Variable Transponder enabled Elastic Optical Networks (SBVT-EON) was rectified. Different traffic grooming strategies were developed separately for achieving various purposes, by varying the edge weights of AG. Then the capacity of a transponder could effectively be utilized by proposing two different techniques for spectrum reservation.

To tackle the increasing issues like traffic demands and the effective use of network resources, optical networks for the next generation were designed by introducing the concept of elasticity in it [11]. The capacity of lightpaths was allowed in a way to fit into the user demands due to the property of elasticity. By employing numerous techniques developed, the resource usage 
could be enhanced further more by means of transmitter or spectrum savings. The usage of transmitters or the number of transmitters were minimized by establishing the optical tunnels which carried numerous numbers of connections in a nearby spectrum block without the insertion of guard bands. Pradhan et al. [15] proposed the new method for reducing the number of requests blocked in dynamic multicast optical networks by reducing the total number of resources like wavelengths, transceivers and splitters which were utilized by the requests and the utilization of bandwidth was also increased at the same time as well. Bin packing problem was considered to be a significant problem in computational complexity theory. For providing effective utilization of resources, two heuristic algorithms such as Multicast Traffic Grooming with Bin packing Best-Fit (MTG-BBF) and Multicast Traffic Grooming with Bin packing First-Fit (MTG-BFF) were proposed in the research paper. The aforementioned heuristic algorithms were developed based on the bin pack heuristic approach.

The heuristic light tree dependent methodology which had been developed known by the name, 0/1 knapsack dependent multi-cast grooming of traffic [14]. They devised this methodology for reducing the cost incurred in networking by making sure of limited counts of several layered optical and electronic components such as splitters, transmitters, and receivers along with the consumed wavelengths while networking. ILP-Integer Linear Programming was deployed by this work for optimizing the cost of the working. An arrangement was suggested for the System with the use of a sliceable optical transponder in order to redress the issues experienced in the traffic grooming using Spectrum Engineering [21]. Effectiveness in terms of bandwidth was targeted the most by this work by developing the whole arrangement based on the wavelength and time multiplex operation. This work held to be good whenever network efficiency was to be maintained in the cases of excessive traffic authorization. The issue of traffic routing and grooming in WDM mesh networks [19] was studied with the aid of the simple path constraint which was indicated as GR. The analysis of the simple path constraint was given as novelty in the research work. Due to the implementation of quadratic constraints by this non-trivial type of simple path constraints, the problem of traffic grooming or routing became a more challenging process. A Greedy Randomized Adaptive Search Procedure (GRASP) was proposed in the work for rectifying the GR problem, by providing a separate mathematical formula for the problem of GR. A total of 38 instances which were three sets, were generated based on the real-world situations within and were applied to GRASP algorithm and the performance.

A novel BAT algorithm for lightpaths routing and spectrum assignment (RSA) [6] which balanced the security level of the domain was proposed. This proposed BAT algorithm was considered to be the evolutionary algorithm as it could be able to route the wavelength assignment and was also employed for the global optimization. The optimization functions based on the behavior of bats using the echolocation character of micro balls that ranging from pulse rate of emission and loudness. The most significant parameters that contributed to the Quality of Services of the optical network was analyzed. The behavior of the nature had effectively been utilized with the heuristics to solve various optimization problem. The behavior of Glowworm is applied to reduce the interference level and optimize the node selection process so as to improvise throughput and Energy Efficient Data Delivery [13]. The Optimization of maritime search and rescue operation was carried out using Ant behavior in the process of searching and pheromone concentration updating strategy of the Ant Colony Algorithm [23]. The improved feature selection, using forest tree nature [20] was carried out with an objective of optimizing the intermediate and subset classifier's classification accuracy by employing Initialization, Selection and Updating process. Various multi-objective parameters like the hop count, bandwidth, wavelength assignment, and throughput were taken into consideration for the monitoring and improving the performance of present networks [7]. The parameters mentioned were responsible for achieving the QoS. The impact of these parameters was explained along with the requirement of the performance of the proposed work. These parameters were assessed in a detailed manner and the usage of these parameters in several research studies were also interpreted. Optimal use of network resources and survivability of data was contributed by the allotted path protection provided and traffic grooming strategies [18]. Assis et al. utilized the mixed-integer linear programming [1] with heuristic and meta-heuristic approaches which 
was used for the virtual optical networks (VONs) design. Traffic adjustments were allowed within each VON, and a Distance adaptive modulation method was used to ensure optimal performance focusing on physical substrate bandwidth. Ruijie et al. [16] proposed the Distance Adaptive Energy Aware Resource Allocation (DERA) algorithm using a multipath survival Method. The DERA algorithm used the concept of Spectrum Compactness (SC) to reduce the powerful effects of crosstalk on Elastic Optical Network while allocating resources. The aim is to save energy consumption, while loading the variable traffic peaks.

\section{The Proposed Work}

The overall flow involved in the proposed Visibility Graph based Iterative Hungarian Traffic Grooming algorithm is illustrated in Figure 2. If the link between the nodes were not at fault, the best k-shortest path was selected by the multi-objective particle swarm optimization. If the link between the nodes was at fault, the next best fit path to forward the data was obtained. After the generation of $k$-shortest paths, the graph was constructed based on the visibility of connected paths. The weights were calcu-

\section{Figure 2}

Overall Flow of the proposed work

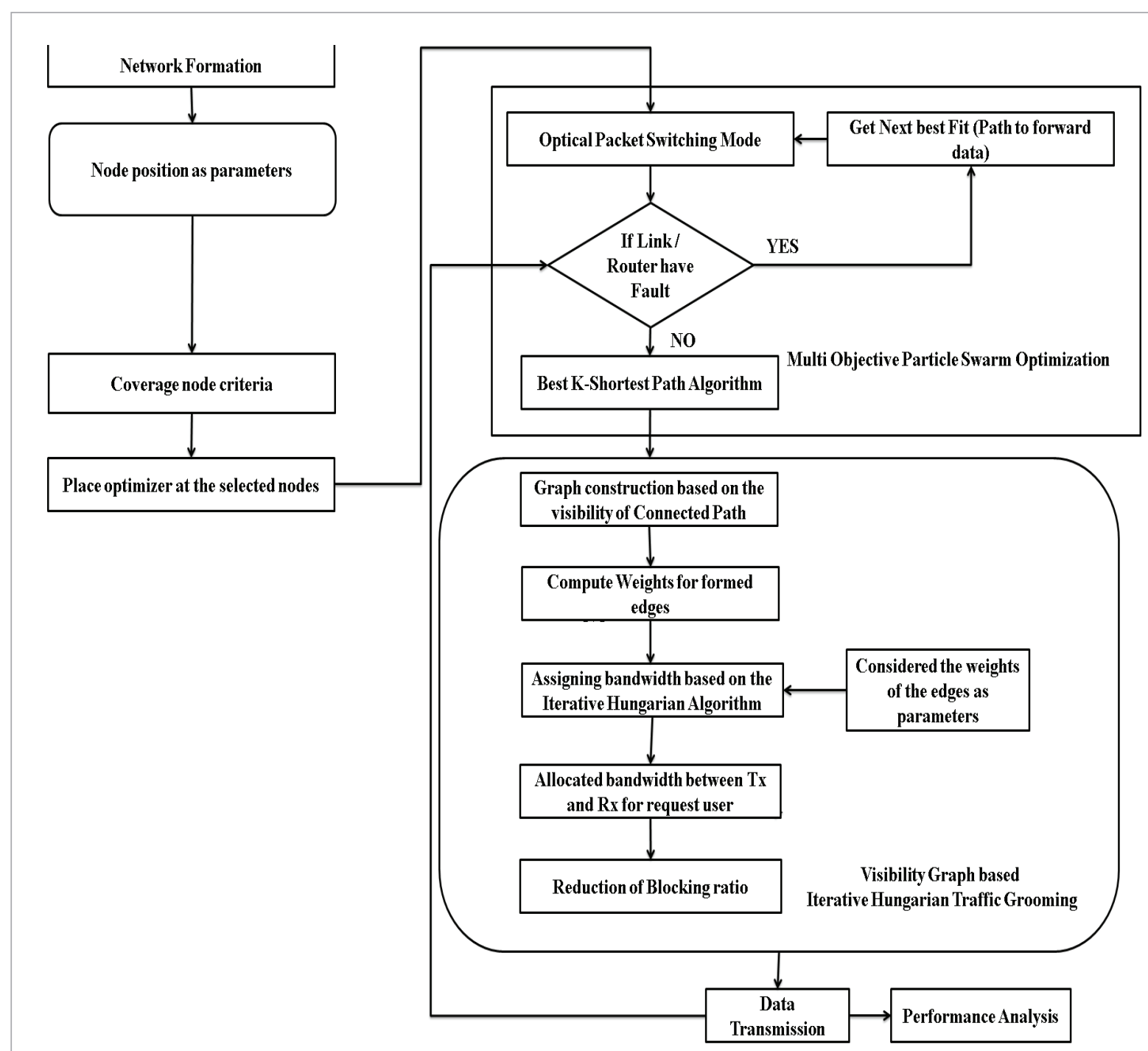


lated separately for the formed edges. Then by considering the weights of the edges to be parameters, the bandwidth was assigned through the Iterative Hungarian Algorithm.

The assigned bandwidth was allocated between the Transmitter and Receiver and by improving the allocation of bandwidth among the users, the blocking ratio could be reduced. After the reduction of blocking ratio, the data transmission was processed among various nodes without any issues. Then the performance of the proposed Visibility Graph based Iterative Hungarian Traffic Grooming algorithm is evaluated by means of various performance metrics like traffic throughput, transceivers count, average propagation delay, blocking ratio, and success ratio.

Algorithm I: Optimizer placement based on coverage criteria

1. Estimate Voronoi diagram through the position of node.

2. Compute interest points for prediction of node connectivity based on the vertices of polygon within $R e$ gion of Interest (ROI), which distributes the interest points beside boundary evenly.

for each IP

$/ / I P$-interest points

$/ / \mathrm{D}$ - distance between interest points and nearest nodes.

$$
\mathrm{D}=\sqrt{\left(\mathrm{IP}_{\mathrm{x}}-\mathrm{N}_{\mathrm{x}}^{\mathrm{i}}\right)^{2}+\left(\mathrm{IP}_{\mathrm{y}}-\mathrm{N}_{\mathrm{y}}^{\mathrm{i}}\right)^{2}}
$$

$/ / \mathrm{r}_{\text {node }}$ - node connectivity

$\mathrm{C}_{\mathrm{IP}}=\{\mathrm{Nx}\}$

$/ / C_{I P}-$ node connectivity for the interest point node

$/ / N_{x}$-node which are presented less than the connectivity

The percentage of node connectivity was maximized, by positioning the nodes in the ROI and this was considered to be the node connectivity problem. The set of $\mathrm{N}$ number of nodes, where $\mathrm{S}=\{\mathrm{s} 1, \mathrm{~s} 2, \ldots ., \mathrm{sN}\}$ and $\mathrm{a}$ ROI are given.

The solution that had been encoded in a particle was evaluated using the fitness function. The maximization problem cited here was the node connectivity problem and maximizing the total area involved in the node connectivity region was considered as the primary objective. By utilizing the Voronoi diagram, the value of the node connectivity could be evaluated. The Voronoi diagram was constructed based on the position of the nodes encoded in a particle. Selection of a set of points known as interest points played a major role in the measurement of nodes region. A number of points which were evenly distributed in the boundary of the polygons and the vertices of the Voronoi polygons were collectively present in the set of interest points. The nodes were prevented from gathering around a particular point in the ROI by utilizing the points present in the boundary which acted as the pulling forces. Selection of the number of points at the boundary was carefully carried out as the presence of numerous points would place the nodes at the very corner of the boundary and similarly a very few points present in the boundary were allowed for the nodes to gather. The entire area of node connectivity region was estimated as follows: The interest point distance to the adjacent nodes was evaluated. If the distance (d) was greater than the connectivity of nodes (cn), then there is a node connectivity region around the point of interest. If the point of interest was a Voronoi vertex, the location of the region was approximated as the circular area around the vertex that was not protected by the nearest detector, while it was a quarter of the circle if it was a corner point on the boundary. while it was on the edge, it would be about half the ring. The node connectivity region was estimated. The distance between $d$ and cn (d-cn) was the connectivity of the node connectivity region circle. The fitness was therefore the summation of the exposure gaps region in the ROI. The fitness value would preferably be equal to zero, implying that there were no gaps in the node connectivity. Assuming there were $\mathrm{K}$ points of interest-boundary points and vertices of Voronoi, it would be possible to compute the fitness function.

\subsection{Detection of Link Failure and the Best Shortest Path}

The path failure and the best shortest paths among the nodes are performed by a multi-objective modified Particle Swarm Optimization algorithm. The algorithm for the same is provided in Algorithm II: 
Algorithm II: Multi-Objective Modified Particle Swarm Optimization

Input: nodes node $e_{i}$ nodes position node $e_{\text {coor }}^{i}$

Output: best shortest paths path $_{\mathrm{i}}^{\mathrm{G}_{\text {best }}}$

Step 1: Initialize particles:

Consider the number of nodes as the particles number,

$P_{i}=$ node $_{i}$

Let take nodes position as the particle's positions,

Pos $_{i}=$ node $_{\text {coor }}^{i}$

The velocity of the particles is initialized random$l y, \mathrm{~V}_{i}=$ rand.

Step 2: Multi Objective Fitness function:

Objective 1:

- Distance estimation between two nodes,

$$
\mathrm{Dij}=\sqrt{\left(\operatorname{Pos}_{\mathrm{i}}-\mathrm{Pos}_{\mathrm{j}}\right)^{2}}
$$

- Weights between two adjacent nodes are estimated,

$$
\mathrm{w}_{\mathrm{ij}}=\frac{\mathrm{D}_{\mathrm{ij}}}{\mathrm{i}^{*} \mathrm{j}}
$$

- Estimate and update shortest path of all links which are adjacent to node ' $i$ ',

$$
\operatorname{path}_{i}^{\text {present }}=\min _{j \rightarrow 1 \text { to } n}\left(D_{i j} W_{i j}+c(i, j)\right)
$$

$\mathrm{C}(\mathrm{i}, \mathrm{j})$ is the constant.

\section{Objective 2:}

- Find the path failure based on the blocking probability,

$$
\begin{aligned}
& \mathrm{PF}_{\mathrm{i}}=\text { Block probability }\left(\text { path }_{\mathrm{i}}^{\text {present }}\right) \\
& \text { path }_{\mathrm{i}}^{\text {present }_{1}}=\operatorname{delete}\left(\text { path }_{\mathrm{i}}^{\text {present }}, \mathrm{PF}_{\mathrm{i}}\right)
\end{aligned}
$$

// delete the failure path from the list of selected shortest path

Step 3: Update the best particle positions and their velocity.

Step 4: Repeat the above steps for prediction of the shortest path between two nodes.

$$
\text { path }_{i}^{\mathrm{G}_{\text {best }}}=\text { path }_{\mathrm{i}}^{\text {present }_{1}}
$$

The particles are initialized at the first step only after considering the number of nodes that are to be the particle numbers. The velocities of the function were randomly initialized. Then, by using the multi objective fitness functions, the distance between two nodes is determined followed by the evaluation of weights between the two adjacent nodes. Then, the shortest path of all links is estimated and updated. Next, the path failure based on the blocking probability is determined. The best particles are updated along the particle position and velocity. Finally, the above steps for prediction of the shortest paths between two nodes are repeated.

\subsection{Dynamic Traffic Grooming}

Various policies are employed for assigning the weight by which the request blocking ratio can be reduced. Reducing the request blocking ratio is considered to be the significant objective that can be achieved with the help of the sufficient amount of available network resources that include a transmitter at the source node, a receiver at the destination node and sufficient bandwidth along the shortest route. When the traffic existing in the same node on the same lightpath is increased or dropped, then a transmitter or a receiver gets shared by the multiple connections. Similarly, the traffic present in the connection request can be added or dropped by allocating a free transmitter or receiver to a new connection request in some of the cases. The connection requests are provisioned for reducing the request blocking ratio by taking the two important categories of resources into consideration. By using the Visibility Graph based Iterative Hungarian Traffic Grooming algorithm, the bandwidth is allocated. Therefore, the edges present in the visibility graph is equivalent to the bandwidth available and the free receivers or transmitters which are assigned above the weights apart from the types of edges.

\subsection{Visibility Graph}

Visibility graph is formed in the first step. The existing light trail is not present in the visibility graph at the initial stage. The visibility graph is implemented with the possible candidate lightpath edges. Graph and edges are formed by analysing the visibility between two nodes. 


$$
e_{i j}=\left\{\begin{array}{c}
1 \quad x_{a}<x_{b} \wedge x_{k}<x_{b} \quad \forall k \in(i, j) \wedge i<j \\
0 \quad \text { otherwise }
\end{array}\right.
$$

Where, $\mathrm{X}_{\mathrm{a}}$ is the source node, $\mathrm{X}_{\mathrm{b}}$ is the destination node and $x_{k}$ are the intermediate nodes.

Then the initialization of hop length paths that are very marginal related to every candidate trail edge are performed. Then different edges like candidate dropping and candidate adding edges are added to the visible graph. When compared to the weight present in different types of edges like candidate adding edge or candidate dropping edge, the value of weight in a grooming edge is very small, especially the weights present in all grooming edges are found to be very small. It can be proven that the significance of a candidate adding edge or the candidate dropping edge is certainly not overshadowed by the grooming edges as the total of the weights present in every grooming edge besides a single lightpath is very small when compared with the weight existing in a candidate adding edge or candidate dropping edge. Then every edge weight is calculated based on the formula (6) given below.

$$
w_{i j}=\left\{\begin{array}{lll}
\left|\left(x_{i}-x_{j}\right)(i-j)\right|+1 & e_{i j} & \text { exists } \\
0 & & \text { otherwise }
\end{array}\right.
$$

The strength of paths is analyzed by utilizing the weights which are formed along the paths.

\subsection{Iterative Hungarian Traffic Grooming}

When communication applications arrive and leave, light trails are increased or decreased to a lower value. The existing lightpath is tested for determining whether it possesses acceptable residual bandwidth, by the arrival of a new link request with the origin node $\mathrm{s}$, destination node $\mathrm{d}$, and bandwidth requirement b. Both prevailing light paths are eliminated from the visible graph without ample residual bandwidth.

A square matrix is constructed from the weights and edges of the nodes. light paths are eliminated from the visible graph without ample residual bandwidth. A square matrix is constructed from the weights and edges of the nodes.

$$
\mathrm{w}_{\mathrm{ab}}^{\mathrm{j}}=\left[\begin{array}{ccc}
\mathrm{W}_{12} & \cdots & \mathrm{w}_{\mathrm{a} 1} \\
\vdots & \ddots & \vdots \\
\mathrm{w}_{1 \mathrm{~b}} & \cdots & \mathrm{w}_{\mathrm{ab}}
\end{array}\right],
$$

where, $\mathrm{w}_{\mathrm{ab}}^{\mathrm{j}}$ is the matrix of $\mathrm{j}^{\text {th }}$ path formed between $a^{\text {th }}$ and $b^{\text {th }}$ node.

$$
\begin{aligned}
& \mathrm{w}_{\mathrm{ab}}=\left[\gamma * \frac{(\operatorname{maxL}(\mathrm{s}, \mathrm{d})-\mathrm{L}(\mathrm{s}, \mathrm{d})}{\mathrm{L}(\mathrm{s}, \mathrm{d})} * \mathrm{Th}\right]+ \\
& {\left[(1-\gamma) *\left(1-\frac{\lambda_{\text {total }}-\lambda(\mathrm{s}, \mathrm{d})}{\lambda(\mathrm{s}, \mathrm{d})}\right) * \mathrm{Th}\right]}
\end{aligned}
$$

where, $w_{a b}$ is the weight value obtained between $a^{\text {th }}$ and $b^{\text {th }}$ node based on the estimation of the throughput.

The weight value is calculated based on the Wavelength Assigned, end to end delay, Gamma factor $(\gamma=1)$ and Throughput. The weight value is calculated for each link and it is updated in the square matrix.

The smallest number of the bandwidth existing in each row is subtracted from each entry in that row.

$$
\mathrm{t}=\left[\begin{array}{c}
\mathrm{w}_{1 \mathrm{n}}^{\prime} \\
\mathrm{w}_{2 \mathrm{n}}^{\prime} \\
\vdots \\
\mathrm{w}_{\mathrm{bn}}^{\prime}
\end{array}\right]
$$

$$
\mathrm{t}_{1}=\left[\mathrm{w}_{\mathrm{b}}^{\prime \prime}\right] \text {. }
$$

Assign all zeros with the minimum number of bandwidths along with the paths and replace zeros over rows and columns using the smallest number of bandwidths for the paths possible to cover all zeros in the matrix.

$$
\mathrm{w}_{\mathrm{ab}}^{\mathrm{j}}=\text { find }\left(\left[\begin{array}{ccc}
\mathrm{w}_{12} & \cdots & \mathrm{w}_{\mathrm{a} 1} \\
\vdots & \ddots & \vdots \\
\mathrm{w}_{1 \mathrm{~b}} & \cdots & \mathrm{w}_{\mathrm{ab}}
\end{array}\right]==\mathrm{t}_{1}\right)
$$

Then the smallest number of the bandwidth in each column is subtracted from each entry in that column. Iterate the above steps until the number of available shortest paths are equal to the number of rows in the square matrix.

Find the strengthen bandwidth that is covered by requested users. 


$$
\left.\begin{array}{l}
\mathrm{bw}^{\mathrm{j}}= \\
\max _{\mathrm{j} \rightarrow 1 \text { to } \mathrm{m}}\left\{\sum_{\mathrm{a}=1}^{\mathrm{n}} \sum_{\mathrm{b}=1}^{\mathrm{n}} \mathrm{w}_{\mathrm{ab}}^{1}, \sum_{\mathrm{a}=1}^{\mathrm{n}} \sum_{\mathrm{b}=1}^{\mathrm{n}} \mathrm{w}_{\mathrm{ab}}^{2}, \ldots, \sum_{\mathrm{a}=1}^{\mathrm{n}} \sum_{\mathrm{b}=1}^{\mathrm{n}} \mathrm{w}_{\mathrm{ab}}^{\mathrm{m}},\right.
\end{array}\right\} .
$$

$b w_{j}$ - represents the path which contains multiple available bandwidths.

Then finally the bandwidth for the request users is assigned.

\section{Performance Analysis}

The proposed system evaluates performance metrics such as traffic throughput, transceivers count, average propagation delay, blocking ratio, and success ratio. Maximum concurrent flow with constraint shortest path as a subroutine (MCF-CSP) [2] aims at providing guaranteed performance and improvised throughput, whereas wind driven optimization (WDO) [5] provides a cost-effective solution for the improvised performance of optical networks. Both of these algorithms in different perspectives prove to show superior quantitative results. Hence, they are taken for comparative evaluation to analyze performance metrics of the proposed algorithm.

\subsection{Traffic Throughput}

This specifies the total traffic that is routed to the digital topology demand for low-speed traffic.

Traffic Throughput(F1)=Max $\sum_{\mathrm{p}=1}^{|\mathrm{N}|} \sum_{\mathrm{q}=1}^{|\mathrm{N}|} \sum_{\mathrm{t}-1}^{\mathrm{A}_{\mathrm{pq}}}\left(\mathrm{x} * \mathrm{~S}_{\mathrm{pq}}^{\mathrm{x}, \mathrm{t}}\right)$.

The Figure 3 shows the throughput of network traffic. The value of throughput shows superior result when comparing it with the MCF-CSP [2] and WDO [5].

Figure 4 represents the comparison of network throughput for different Network Load. The value of network throughput yielded by the proposed algorithm is compared with the existing network throughput respectively when performed with 70 nodes topology as followed in [9].

The Figure 5 represents the comparison of network throughput for different Network Load. The value of network throughput yielded by the proposed algorithm is compared to the existing network throughput
Figure 3

Traffic Throughpu

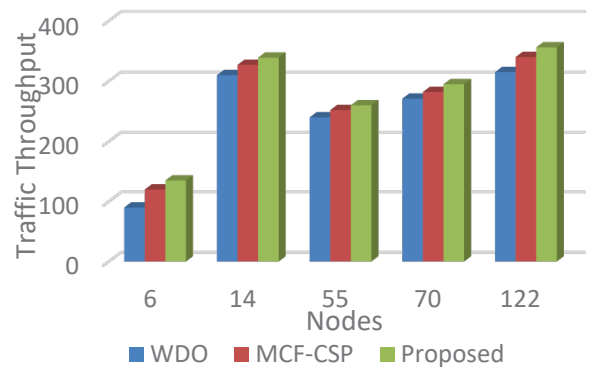

Figure 4

Network Throughput at 70 nodes

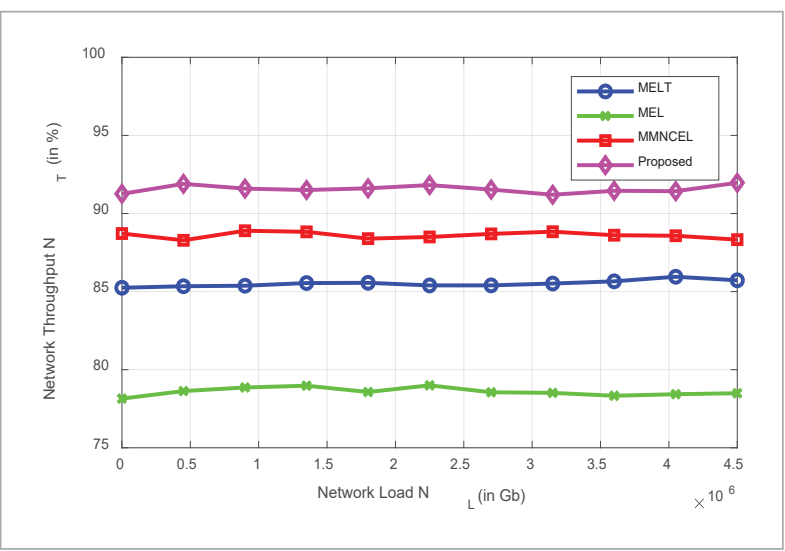

Figure 5

Network Throughput at 122 nodes

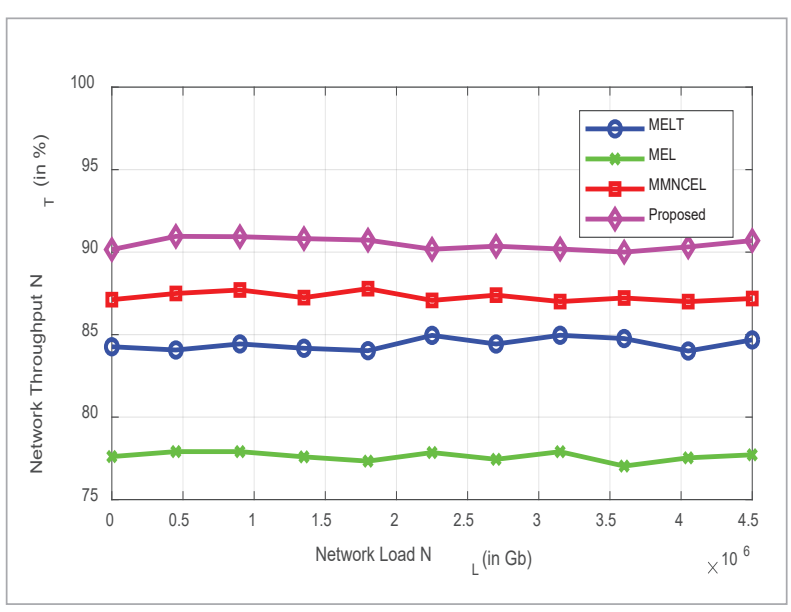

respective when performed with 122 nodes topology as followed in [9]. 


\subsection{Number of Transceivers Count}

This specifies the total number of transceivers or a total number of lightpaths produced in transmission.

Transceivers count $(\mathrm{F} 2)=\operatorname{Min} \sum_{\mathrm{r}=1}^{|\mathrm{N}|} \sum_{\mathrm{s} 1}^{|\mathrm{N}|} \sum_{\mathrm{w}=1}^{\mathrm{A}_{\mathrm{pq}}^{\mathrm{X}}} \mathrm{V}_{\mathrm{pq}}^{\mathrm{W}}$ (14)

The Figure 6 indicates the total number of transceivers count. The count of transceivers is more, while comparing it with the MCF-CSP [2] and WDO [5].

\subsection{Average Propagation Delay: (APD, F3)}

The average number of lightpaths produced gets re-

Figure 6

Transceiver's count

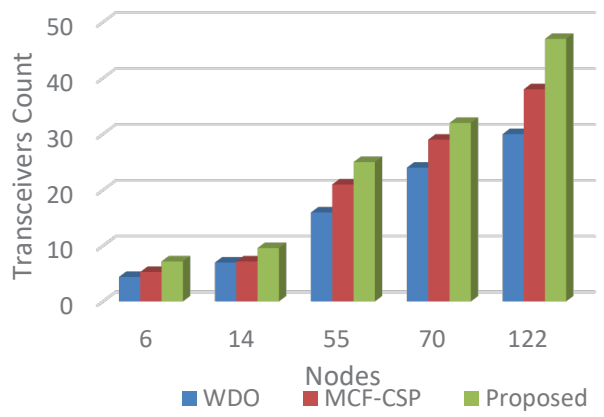

duced by the usage of objective function that assumes full links below.

$$
\begin{gathered}
\mathrm{d}_{\mathrm{kl}}=1 \\
\mathrm{~F} 3=\min \sum_{\mathrm{r}=1}^{|\mathrm{N}|} \sum_{\mathrm{s}=1}^{\mathrm{N}} \sum_{\mathrm{k}=1}^{\mathrm{N}} \sum_{\mathrm{l}=1}^{\mathrm{N}}\left(\mathrm{d}_{\mathrm{k} l} * \sum_{\mathrm{w}=1}^{\mathrm{N}} \mathrm{P}_{\mathrm{kl}}^{\mathrm{r}, \mathrm{s}, \mathrm{w}}\right) / \sum_{\mathrm{r}=1}^{|\mathrm{N}|} \sum_{\mathrm{s} 1}^{|\mathrm{N}|} \sum_{\mathrm{w}=1}^{\mathrm{w}} \mathrm{V}_{\mathrm{r}, \mathrm{s}}^{\mathrm{w}} \cdot
\end{gathered}
$$

The fitness function is evaluated as,

$\mathrm{F}=\min (\mathrm{F} 1-\mathrm{F} 2-\mathrm{F} 3)$

Figure 7 represents the average propagation delay. The delay of the proposed algorithm is reduced while comparing it with the MCF-CSP [2] and WDO [5].

\subsection{Blocking Ratio}

The ratio of number of the connection requests that are blocked to sum of the connection requests is termed as blocking ratio. The Blocking ratio is considered to be the important parameter in determining the QoS.
Figure 7

Average propagation delay

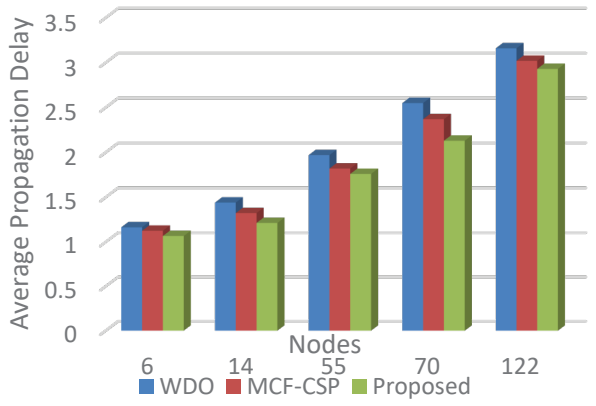

Figure 8 a) represents the Request blocking ratio for different Arrival Rate with the scenario that the numbers of transmitters and receivers at each node are $75 \%$ of maximum required number and Figure $8 \mathrm{~b}$ ) represents the Request blocking ratio for different $\mathrm{Ar}$ -

Figure 8

Request blocking ratio

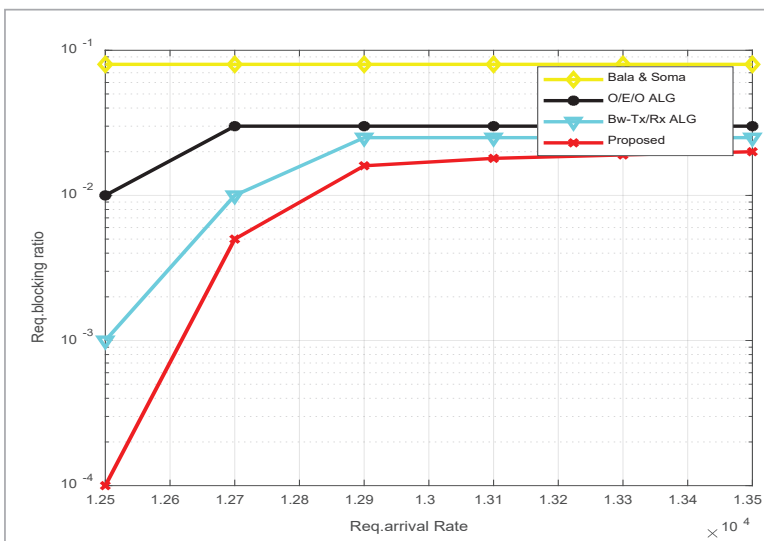

(a)

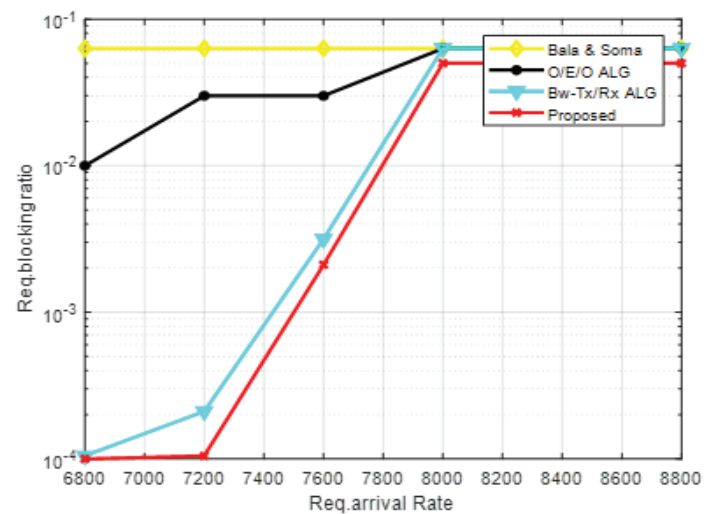

(b) 
rival Rate when the numbers of transmitters and receivers at each node are reduced to $25 \%$ of maximum required numbers. The proposed algorithm is compared with the existing algorithms and it is inferred that the proposed algorithm consists of less blocking ratio while comparing it with the other existing algorithms since the bandwidth resources are utilized conservatively. Thus, the increase in the transmitter and receiver count allows more free connection for the upcoming new arrival packets thereby reduces blocking probability and utilizes the bandwidth more conservatively and efficiently than other algorithms.

\subsection{Success Ratio}

The ratio of number of packets received to the total number of packets sent is termed as Success ratio.

Figure 9 indicates the value of success ratio. The Success ratio of the proposed algorithm is improved while comparing it to the MCF-CSP [2] and WDO [5].

\section{Conclusion}

The traffic grooming was performed with the aid of the Visibility Graph based Iterative Hungarian Traffic Grooming algorithm. The path failures among the nodes were detected. The best suitable shortest path was determined by the multi objective modified PSO algorithm and the traffic congestion in a network was reduced by the allocation of bandwidths by utilizing an innovative visibility graph based Iterative Hun-

\section{References}

1. Assis, K. D. R., Santos, A. F., Almeida, R. C., Reed, M. J., Jaumard, B., Simeonidou, D. Virtualization of Elastic Optical Networks and Regenerators with Traffic Grooming. IEEE/OSA Journal of Optical Communications and Networking, 2020, 12(12), 428-442. https:// doi.org/10.1364/JOCN.398749

2. Cao, Z., Claisse, P., Essiambre, R.J., Kodialam, M., Lakshman, T. Optimizing Throughput in Optical Networks: The Joint Routing and Power Control Problem. IEEE/ ACM Transactions on Networking (TON), 2017, 25(1), 199-209. https://doi.org/10.1109/TNET.2016.2578321

3. Eira, A., Pedro, J., Pires, J. Traffic Grooming Policies Under Switching Constraints in Next-Generation Transport Networks. Journal of Optical Communica-

\section{Figure 9}

Success ratio

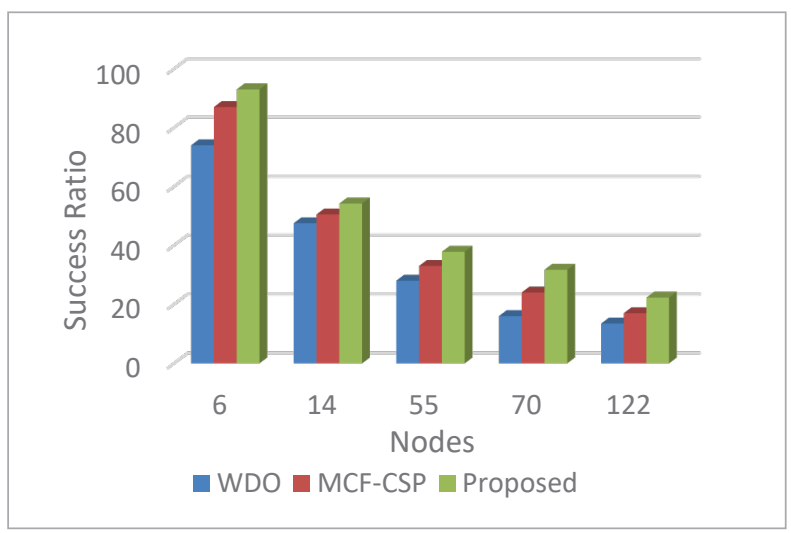

garian Traffic grooming algorithm which was implemented to reduce the blocking ratio by improving the allocation of bandwidth between the users. Finally, the performance analysis was carried out by means of performance measures such as traffic throughput, transceivers count, average propagation delay, blocking ratio, and success ratio. It could be inferred that the proposed work was obtained with enhanced outcomes when compared with the other existing techniques. Compared with other algorithms, it was found that there is an increase in the traffic throughput, transceivers count and success ratio in the proposed algorithm. Besides these, the performance measures such as average propagation delay and blocking ratio of the proposed algorithm were found to be less while comparing it with other algorithms.

tions and Networking, 2017, 9(1), A125-A134. https:// doi.org/10.1364/JOCN.9.00A125

4. Fan, Z., Qiu, Y., Chan, C. K. Dynamic Multipath Routing with Traffic Grooming in OFDM-Based Elastic Optical Path Networks. Journal of Lightwave Technology, 2015, 33(1), 275-281. https://doi.org/10.1109/ JLT.2014.2387312

5. Kaur, H., Rattan, M. Wind Driven Based Heuristic Solution for Multiobjective Traffic Grooming in Optical Networks. Wireless Personal Communications, 2019, 110(3), 1-17. https://doi.org/10.1007//s112777-019-06796-y

6. Kumarnath, J., Batri, K. A BAT Algorithm Based Enhancement of Physical Layer Security in a Muti-Domain-Elastic 
Optical Network. International Journal of Pure and Applied Mathematics, 2018, 119(15), 2519-2525.

7. Kumarnath,J., Batri, K. A Novel Parametric Analysis of the Performance Dynamicity of Optical Network. Advances in Natural and Applied Sciences, 2017, 11(9), 166-174.

8. Lin, H.C., Zhuang, Y. X. A Novel Auxiliary Graph Model for Effective Dynamic Traffic Grooming in Light-Trail Optical WDM Mesh Networks. Photonic Network Communications, 2019, 38(1), 1-13. https://doi.org/10.1007/ s11107-019-00837-z

9. Majumdar, P., De, T. De-Multiplexing the Required Spectrum in a Traffic Demand Into Multiple Non-Adjacent Granular Spectrums for Dynamic Traffic Grooming in EON. Optical Switching and Networking, 2019, 33, 143-160. https://doi.org/10.1016/j.osn.2018.04.001

10. Majumdar, P., Pal, A., De, T. Extending Light-Trail Into Elastic Optical Networks for Dynamic Traffic Grooming. Optical Switching and Networking, 2016, 20, 1-15. https://doi.org/10.1016/j.osn.2015.10.005

11. Miladic-Tesic, S., Markovic, G., Radojicic, V. Traffic Grooming Technique for Elastic Optical Networks: A Survey. Optik, 2019, 176, 464-475. https://doi. org/10.1016/j.ijleo.2018.09.068

12. Panayiotou, T., Ellinas, G., Antoniades, N. Hybrid Graph-Based Multicast Traffic Grooming in Metro Networks with Quality-of-Transmission Considerations. Photonic Network Communications, 2016, 32(1), 142159. https://doi.org/10.1007/s11107-015-0589-9

13. Perumal, T., Subramaniyan, S. Statistical Markov Model Based Natural Inspired Glowworm Swarm Multi-Objective Optimization for Energy Efficient Data Delivery in MANET. Information Technology and Control, 2020, 49(2), 333-347. https://doi.org/10.5755/j01.itc.49.2.23554

14. Pradhan, A. K., Chatterjee, B. C., Oki, E., De, T. Knapsack Based Multicast Traffic Grooming for Optical Networks. Optical Switching and Networking, 2018, 27(C), 40-49. https://doi.org/10.1016/j.osn.2017.08.002

15. Pradhan, A. K., Singhi, S., De, T. Multicast Dynamic Traffic Grooming Using Bin Packing Method in WDM Mesh Networks. Optical Switching and Networking, 2017, 23(1), 40-51. https://doi.org/10.1016/j.osn.2016.08.004

16. Ruijie, Z., Aretor, S., Peisen, W., Shihua, L., Lulu, L., Pei, L., Mingliang, X. Survival Multipath Energy-Aware Re- source Allocation in SDM-EONs During Fluctuating Traffic, Journal of Lightwave Technology, 2021, 39(7), 1900-1912. https://doi.org/10.1109/JLT.2020.3043271

17. Sankaran, G. C., Sivalingam, K. M. Optical Traffic Grooming-Based Data Center Networks: Node Architecture and Comparison. IEEE Journal on Selected Areas in Communications, 2016, 34(5), 1618-1630. https:// doi.org/10.1109/JSAC.2016.2520214

18. Soheil, H., Akbar, G. R, Mohammad, J. B.Survivable Time-Aware Traffic Grooming in Spatial Division Multiplexing Elastic Optical Networks with Minimized Crosstalk. Computers and Electrical Engineering, 83(11), 2020, 1 -15. https://doi.org/10.1016/j.compeleceng.2020.106579

19. Wu, X., Ye, T., Guo, Q., Lü, Z. GRASP for Traffic Grooming and Routing with Simple Path Constraints in WDM Mesh Networks. Computer Networks, 2015, 86, 27-39. https://doi.org/10.1016/j.comnet.2015.04.013

20. Xie, Q., Cheng, G., Zhang, X., Lei, P. Feature Selection Using Improved Forest Optimization Algorithm. Information Technology and Control, 2020, 49(1), 289-301. ttps://doi.org/10.5755/j01.itc.49.2.24858

21. Yu, X., Zhao, Y., Zhang, J., Wang, J., Zhang, G., Chen, X., Zhang, J. Dynamic Traffic Grooming with Spectrum Engineering (TG-SE) in Flexible Grid Optical Networks. Optical Fiber Technology, 2015, 26, 150-156. https://doi. org/10.1016/j.yofte.2015.10.001

22. Zhang, J., Ji, Y., Song, M., Zhao, Y., Yu, X., Zhang, J., Mukherjee, B. Dynamic Traffic Grooming in Sliceable Bandwidth-Variable Transponder-Enabled Elastic Optical Networks. Journal of Lightwave Technology, 2015, 33(1), 183-191. https://doi.org/10.1109/JLT.2014.2383444

23. Zhang, H., Sun, J., Yang, B., Shi, Y., Li, Z. Optimal Search and Rescue Route Design Using an Improved Ant Colony Optimization. Information Technology and Control, 2020, 49(3), 438-447. https://doi.org/10.5755/j01. itc.49.3.25295

24. Zhao, Y., Tian, R., Yu, X., Zhang, J., Zhang, J. An Auxiliary Graph Based Dynamic Traffic Grooming Algorithm in Spatial Division Multiplexing Enabled Elastic Optical Networks with Multi-Core Fibers. Optical Fiber Technology, 2017, 34, 52-58. https://doi.org/10.1016/j. yofte.2017.01.005 